\title{
Demanda hídrica e coeficientes de cultura (Kc) para macieiras em Vacaria, RS
}

\author{
Water requirement and crop coefficients (Kc) for apple trees in Vacaria, RS, Brazil
}

\author{
Marco Antônio Fonseca Conceição ${ }^{\mathrm{I}}$ Gilmar Ribeiro Nachtigall ${ }^{\mathrm{II}}$

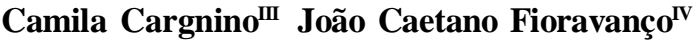

\begin{abstract}
O objetivo do presente trabalho foi determinar a demanda hídrica e os coeficientes de cultura (Kc) para macieiras cultivadas na região de Vacaria, RS. O trabalho foi desenvolvido na Estação Experimental de Fruticultura Temperada (EEFT) da Embrapa Uva e Vinho, localizada em Vacaria, RS, em plantas da cultivar 'Royal Gala' (Malus domestica) sobre porta-enxerto M9. Foram determinados os valores do potencial matricial da água no solo, empregandose tensiômetros de punção. Com base nesses valores, determinou-se a umidade volumétrica e o balanço hídrico mensal. O consumo hídrico da cultura variou entre $0,3 \mathrm{~mm}$ dia $^{-1}$ a 4,5mm dia ${ }^{-1}$, com média de $1,9 \mathrm{~mm} \mathrm{dia}^{-1}$. O coeficiente da cultura $(K c)$ apresentou tendência quadrática, variando entre 0,19 e 0,88, com média igual a 0,58.
\end{abstract}

Palavras-chave: maçã, Malus domestica, evapotranspiração.

\section{ABSTRACT}

The objective of this study was to determine the water requirement and the crop coefficients $(K c)$ values for apple trees cultivated in Vacaria, RS. The study was conducted at the Experimental Station of Temperate Fruits (EEFT) of Embrapa Grape and Wine, located in Vacaria, RS, in plants of the cultivar 'Royal Gala' (Malus domestica) on the rootstock M9. The soil water matric potential values, that were determined employing tensiometers, were used to obtain the soil moisture and the monthly soil water balance. The water consumption of the culture ranged from $0.3 \mathrm{~mm}$ day-1 to $4.5 \mathrm{~mm}$ day-1, with an average of $1.9 \mathrm{~mm} \mathrm{day}^{-1}$. The crop coefficient $(\mathrm{Kc})$ presented a quadratic trend, ranging between 0.19 and 0.88 , with mean value of 0.58 .

Key words: apple, Malus domestica, evapotranspiration.
O cultivo da macieira tem se expandido na região sul do Brasil, que apresenta, atualmente, mais de 30.000ha com a cultura (MELLO et al., 2007). Embora as principais regiões produtoras apresentem elevados índices pluviométricos, é comum a ocorrência de déficit hídrico durante o ciclo da cultura, principalmente no final da primavera e durante o verão, o que pode prejudicar a qualidade e a produtividade comercial. Por essa razão, há uma demanda recente, por parte de produtores da região, pela instalação de sistemas de irrigação em suas áreas. Essa tendência também tem sido observada em outras regiões úmidas do mundo, que são produtoras de maçãs (GARCÍA-PETILLO et al., 2003; DRAGONI et al., 2004).

Para o dimensionamento e manejo da irrigação, são empregados, normalmente, valores do coeficiente da cultura $(\mathrm{Kc})$ na estimativa da demanda hídrica do pomar. Embora existam dados de Kc para macieiras na literatura (ALLEN et al., 2006; GONG et al., 2007), nem sempre esses valores publicados se ajustam às condições locais (DRAGONI et al., 2004). O objetivo do presente trabalho foi, assim, determinar a demanda hídrica e os valores de Kc para macieiras cultivadas na região de Vacaria, RS.

O trabalho foi desenvolvido na Estação Experimental de Fruticultura Temperada (EEFT) da Embrapa Uva e Vinho, localizada em Vacaria, RS (latitude $28^{\circ} 33^{\prime} \mathrm{S}$, longitude $50^{\circ} 57^{\prime} \mathrm{W}$, e $955 \mathrm{~m}$ de

'Embrapa Uva e Vinho, Estação Experimental de Viticultura Tropical, CP 241, 15700-971, Jales, SP, Brasil. E-mail: marcoafc@cnpuv.embrapa.br. Autor para correspondência.

"Embrapa Uva e Vinho, Estação Experimental de Fruticultura Temperada, Vacaria, RS, Brasil.

"IUniversidade de Caxias do Sul (UCS), Campus Vacaria, Vacaria, RS, Brasil.

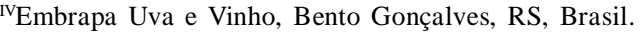


altitude). As avaliações foram realizadas em plantas de nove anos da cultivar 'Royal Gala' (Malus domestica) sobre porta-enxerto M9, conduzidas com líder central, em plantio de alta densidade, espaçadas em $0,8 \mathrm{mx} 3,8 \mathrm{~m}$ $\left(3,04 \mathrm{~m}^{2}\right.$ planta $\left.^{-1}\right)$. O solo foi classificado com Latossolo Bruno Distrófico, apresentando uma capacidade média de água disponível de, aproximadamente, $140 \mathrm{~mm} \mathrm{~m}^{-1}$.

Para o monitoramento hídrico do solo, foram empregados tensiômetros de punção instalados em duas profundidades, 10 e $30 \mathrm{~cm}$, entre três pares de plantas, correspondendo às camadas de $0 \mathrm{a} 20 \mathrm{~cm}$ e de 20 a $40 \mathrm{~cm}$, respectivamente. O intervalo de 0 a $40 \mathrm{~cm}$ é onde se concentra a maior parte do sistema radicular da cultura (GREEN \& CLOTHIER, 1999; HOFFMANN \& BERNARDI, 2004), correspondendo à profundidade efetiva (PE) das raízes, que é o valor empregado nos cálculos de dimensionamento e manejo da irrigação. A partir dos valores do potencial matricial da água no solo, obtidos com a leitura dos tensiômetros, determinou-se a umidade volumétrica, empregando-se a equação de VAN GENUCHTEN (1980).

Os valores da evapotranspiração da cultura (ETc) e dos coeficientes da cultura (Kc) foram determinados entre os dias 20 de novembro de 2008 a 30 de abril de 2009, que corresponderam, respectivamente, aos períodos de crescimento de fruto até o pós-colheita e início da dormência. A evapotranspiração de referência (ETo) foi calculada diariamente empregando-se o modelo de PenmanMonteith (ALLEN et al., 2006), a partir de dados obtidos na estação meteorológica da EEFT, onde também foram coletados os dados da precipitação pluvial.

Os valores de Kc foram ajustados por meio de balanço hídrico mensal, de acordo com modelo apresentado por ALLEN et al. (2006). Os ajustes de Kc foram realizados obtendo-se, através de processo iterativo, os menores erros padrões da estimativa entre os valores do armazenamento da água no solo (ARM), medidos em campo e estimados pelo modelo. Foram considerados excedentes hídricos os valores de umidade na camada de 20 a $40 \mathrm{~cm}$ superiores à capacidade de campo, que correspondeu ao potencial matricial igual a -10kPa (STRECK et al., 2008; REICHERT et al., 2009).

A ocorrência de deficiência hídrica foi computada quando o consumo de água foi maior do que a fração da água do solo facilmente disponível para as plantas (AFD). Os valores de AFD foram determinados multiplicando-se a água disponível para a cultura (AD) pela fração de água facilmente disponível para as plantas (FAFD), que foi calculada em função da evapotranspiração da cultura (ETc), de acordo com a expressão (ALLEN et al., 2006): $\mathrm{FAFD}=0,50+0,04 .(5,00-\mathrm{ETc})(1)$, sendo FAFD adimensional e ETc em mm dia ${ }^{-1}$. O valor de AD foi igual a 50mm, correspondendo à diferença entre a capacidade de campo (CC) e o ponto de murcha permanente (PMP) na profundidade efetiva do sistema radicular.

Durante o período de avaliação, a precipitação pluvial foi de 431,4mm e os valores médios das temperaturas máxima, média e mínima do ar foram iguais a $24,8,18,7$ e $13,7^{\circ} \mathrm{C}$, respectivamente. Esses valores de temperatura ficaram próximos às médias históricas do período (EMBRAPAUVAE VINHO, 2010), mas a precipitação pluvial foi inferior. Entretanto, o déficit hídrico total no período, contabilizado pelo modelo, foi de apenas $16,6 \mathrm{~mm}$. Já o excedente hídrico total foi de $129,6 \mathrm{~mm}$.

A evapotranspiração de referência (ETo) diária oscilou de 1,3 a 6,1 $\mathrm{mm} \mathrm{dia}^{-1}$, com média de 3,5mm dia $^{-1}$. Já os valores médios mensais de ETo variaram entre 2,5 e 4,1 $\mathrm{mm} \mathrm{dia}^{-1}$ (Figura 1). Os períodos de maior demanda evaporativa atmosférica e, consequentemente, com maiores valores médios de ETo, foram o final de novembro e o mês de dezembro. A partir de janeiro, esses valores começaram a cair, sendo que, no mês de abril, a ETo atingiu seu valor mínimo, que foi de $2,5 \mathrm{~mm}$ $\operatorname{dia}^{-1}$ (Figura 1).

O valor total de ETc no período foi de $314,8 \mathrm{~mm}$, com média de $1,9 \mathrm{~mm}$ dia $^{-1}$ e variações diárias entre 0,3 e 4,5mm dia ${ }^{-1}$. As médias mensais de ETc oscilaram de 0,3 a 3,0 $\mathrm{mm} \mathrm{dia}^{-1}$ (Figura 1). Os maiores valores médios de ETc ocorreram nos meses de janeiro, fevereiro e março, coincidindo com os períodos de crescimento e maturação de frutos, colheita e póscolheita. Os menores valores de ETc foram registrados no início e no final do ciclo da cultura (Figura 1), correspondendo aos períodos de início de crescimento dos frutos e início da hibernação, respectivamente. GONG et al. (2007) registraram, na China, valor médio de ETc, igual a 2,2 $\mathrm{mm} \mathrm{dia}^{-1}$, com um máximo de $4,4 \mathrm{~mm}$ dia $^{-1}$, próximos aos observados no presente trabalho. GARCÍA-PETILLO et al. (2003) observaram, no Uruguai, valores máximos de ETc ao redor de 5,0mm.dia ${ }^{-1}$.

$\mathrm{O}$ coeficiente de cultura $(\mathrm{Kc})$ médio mensal apresentou tendência quadrática (Figura 2), variando entre 0,19 e 0,88 , com média igual a 0,58. GONG et al. (2007) também registraram um comportamento quadrático para os valores de $\mathrm{Kc}$, que variaram entre 0,43 e 1,01 .

De acordo com recomendações da FAO (ALLEN et al., 2006), os valores de Kc para a macieira variam entre 0,80 e 1,20 durante o ciclo da cultura, 


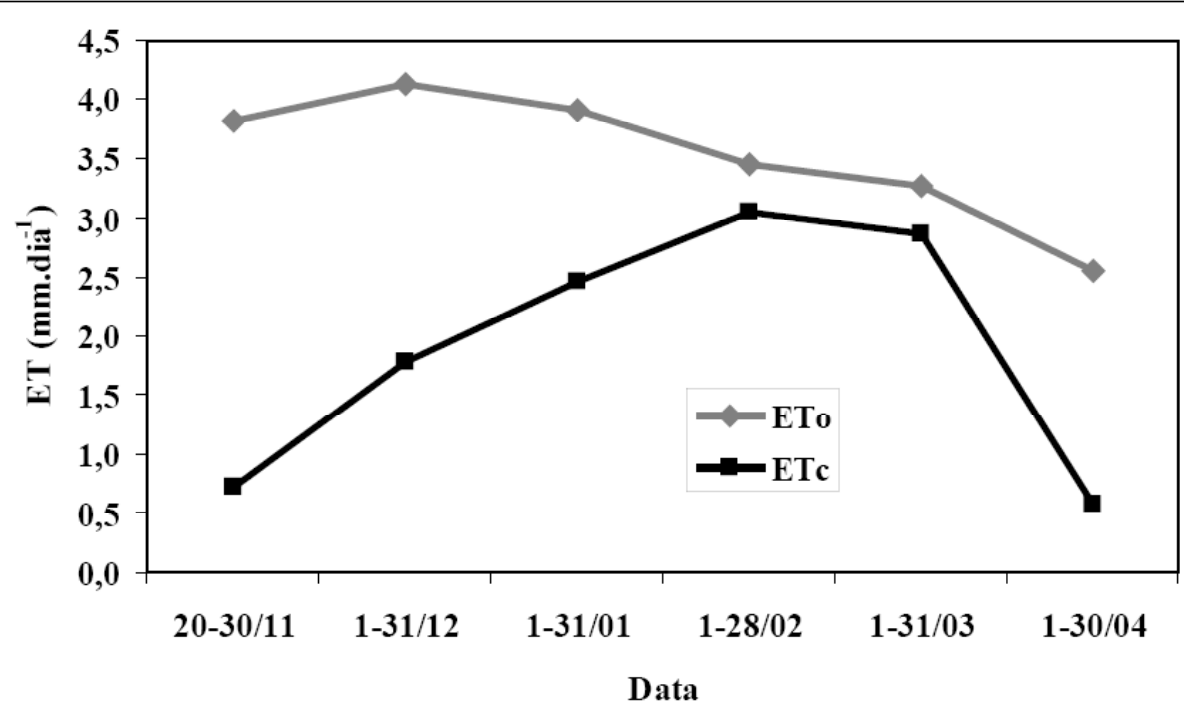

Figura 1 - Valores médios de evapotranspiração de referência (ETo) e de evapotranspiração da cultura (ETc) de macieiras cv. 'Royal Gala'. Vacaria, RS, 2008-2009.

superiores aos observados no presente trabalho. $\mathrm{O}$ uso desses coeficientes nas condições de Vacaria superestimaria, assim, os valores de ETc, proporcionando um desperdício de água e energia, caso fossem utilizados no manejo da irrigação. Daí a importância de se ajustar esses valores às condições específicas de cada local.

\section{AGRADECIMENTOS}

Os autores agradecem à Financiadora de Estudos e projetos (FINEP), à Associação Brasileira de Produtores de Maçã (ABPM) e ao Conselho Nacional de Desenvolvimento Científico e Tecnológico ( $\mathrm{CNPq}$ ), pelo apoio financeiro durante o desenvolvimento do projeto "INOVAÇÕES TECNOLÓGICAS PARA A MODERNIZAÇÃO DO SETOR DA MAÇÃ INOVAMAÇÃ.

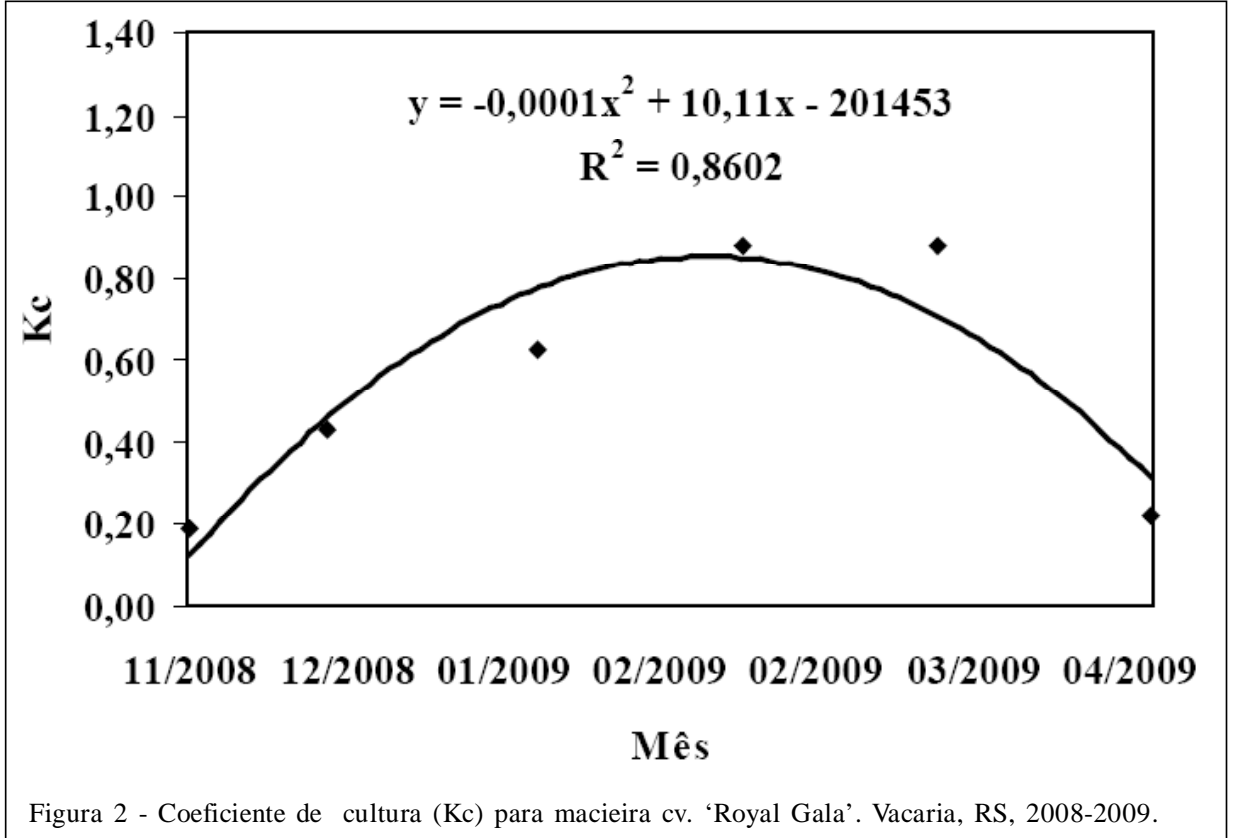

Ciência Rural, v.41, n.3, mar, 2011. 


\section{REFERÊNCIAS}

ALLEN, R. G. et al. Evapotranspiración del cultivo. Guías para la determinación de los requerimientos de agua de los cultivos. Roma: FAO, 2006. 298p.

DRAGONI, D. et al. Transpiration of an apple orchard in a cool humid climate: measurement and modeling. Acta Horticulturae, Wageningen, v.664, p.175-180, 2004. Disponível em: <http:// www.actahort.org/books/664/664_19.htm>. Acesso em: 06 dez. 2010.

EMBRAPA UVA E VINHO. Descrição de local do Sistema CCM Geovitícola. Disponível em: <http://www.cnpuv.embrapa.br/ tecnologias $/ \mathrm{ccm} /$ local.php?regiao=BRcc\&local=1>. Acesso em: 03 nov. 2010 .

GARCÍA-PETILLO, M. et al. Respuesta al riego de duraznero, manzano y peral en montes comerciales. Agrociência, Montevideo, v.7, n.2, p.49-61, 2003. Disponível em: <http:/ /www.fagro.edu.uy/agrociencia/VOL7/2/p49-61.pdf>. Acesso em: 06 dez. 2010.

GONG, D. et al. Estimation of evapotranspiration and its components from an apple orchard in northwest China using sap flow and water balance methods. Hydrological Processes, Chichester, v.21, p.931-938, 2007. Disponível em: <http:// onlinelibrary.wiley.com/doi/10.1002/hyp.6284/pdf>. Acesso em: 06 dez. 2010. doi: 10.1002/hyp.6284

GREEN, S.; CLOTHIER, B. The root zone dynamics of water uptake by a mature apple tree. Plant and Soil, Dordrecht, v.206, p.61-77, 1999. Disponível em: <http://www.springerlink.com/ content/u361685886662r40/fulltext.pdf $>$. Acesso em: 06 dez. 2010. doi: 10.1023/A:1004368906698.

HOFFMANN, A.; BERNARDI, J. Aspectos botânicos. In: NACHTIGALL, G.R. (Ed.). Maçã: produção. Bento Gonçalves: Embrapa Uva e Vinho; Brasília: Embrapa Informação Tecnológica, 2004. p.17-24.

MELLO, L.M.R. de et al. Evolução e dinâmica da produção de maçã no Brasil no período de 1975 a 2003 . Bento Gonçalves: Embrapa Uva e Vinho, 2007. 38p. (Documentos/ Embrapa Uva e Vinho, 66).

REICHERT, J.M. et al. Estimation of water retention and availability in soils of Rio Grande do Sul. Revista Brasileira de Ciência do Solo, Viçosa, v.33, p.1547-1560, 2009. Disponível em: <http://www.scielo.br/scielo.php?pid=S0100$06832009000600004 \&$ script $=$ sci_arttext $\&$ thlng $=e n>$. Acesso em: 06 dez. 2010. doi: 10.1590/S0100-06832009000600004.

STRECK, C.A. et al. Relações do parâmetro S para algumas propriedades físicas de solos do sul do Brasil. Revista Brasileira de Ciência do Solo, Viçosa, v.32, p.2603-2612, 2008. Disponível em: <http://www.scielo.br/scielo.php?pid=S0100$06832008000700001 \&$ script $=$ sci_arttext $\&$ t $\operatorname{lng}=$ en $>$. Acesso em: 06 dez. 2010. doi: 10.1590/S0100-06832008000700001.

Van GENUCHTEN, M.T. A closed-form equation for predicting the hydraulic conductivity of unsaturated soils. Soil Science Society of America Journal, Madison, v.44, p.892-898, 1980. Disponível em: <https://www.soils.org/publications/sssaj/pdfs/44/ 5/SS0440050892 >. Acesso em: 06 dez. 2010. doi: 10.2136/ sssaj 1980.03615995004400050002x. 